\title{
A Longitudinal Study of Trends in Burnout During Primary Care Transformation
}

Kevin Grumbach, $M D^{1}$

Margae Knox, MPH

Beatrice Huang, $B A^{1}$

Hali Hammer, $M D^{2}$

Coleen Kivlaban, MD, MSPH

Rachel Willard-Grace, MPH

${ }^{1}$ Center for Excellence in Primary Care, Department of Family \& Community Medicine, University of California San Francisco, San Francisco, California

${ }^{2}$ San Francisco Health Network and Department of Family \& Community Medicine, University of California San Francisco, San Francisco, California

${ }^{3}$ UCSF Health and Department of Family \& Community Medicine, University of California San Francisco, San Francisco, California
Conflicts of interest: authors report none.

\section{CORRESPONDING AUTHOR}

Kevin Grumbach, MD

UCSF Department of Family \&

Community Medicine

San Francisco General Hospital

1001 Potrero Ave, Ward 83

San Francisco, CA 94110

kevin.grumbach@ucsf.edu

\begin{abstract}
PURPOSE The quadruple aim of primary care transformation includes promoting well-being among the primary care workforce. We longitudinally assessed burnout among clinicians and staff in 2 health delivery organizations engaged in primary care redesign guided by a shared transformation model.
\end{abstract}

METHODS We conducted a descriptive longitudinal study, using repeated crosssectional measures from 6 waves of surveys of employed primary care clinicians (physicians, nurse practitioners, physician assistants) and staff conducted between 2012 to 2018 in the San Francisco Health Network and in UCSF Health. The 2018 wave had 613 respondents (response rate 88\%). Outcome measures were scores on the Maslach Burnout Inventory emotional exhaustion and cynicism subscales. We used regression models to test for time trends in mean scores.

RESULTS Trends in burnout differed by system and occupation. In one system, mean clinician scores steadily improved for emotional exhaustion $(P=.04)$ and cynicism $(P=.07)$. In the other system, clinician burnout scores initially worsened and then returned to baseline levels. In both systems, burnout trends among staff tended to move in the opposite direction from trends among clinicians.

CONCLUSIONS The divergent trends of steady reduction in clinician burnout in one system and clinician burnout getting worse before getting better in the other system suggest that the effects of primary care transformation are influenced by the organizational context. Moreover, practice changes that reduce clinician burnout may not decrease-and may potentially even worsen-burnout among staff. Primary care transformation requires continuing efforts to promote meaningful work and sustainable workloads among all members of the primary care team.

Ann Fam Med 2019;17:S9-S16. https://doi.org/10.1370/afm.2406.

\section{INTRODUCTION}

The prevalence of burnout among US physicians, particularly among family physicians and general internists, is troublingly high. ${ }^{1}$ Crosssectional studies have documented a variety of workplace factors associated with burnout, such as less control over work, lack of teamwork, inadequate resources to address patients' social needs, and the challenges of electronic health records..$^{2-5}$ Much less research, however, has investigated whether changes in the workplace such as primary care practice transformation reduce burnout. Two recent systematic reviews found that the majority of studies of burnout focused on enhancing individuals' resilience rather than changing the conditions of work, and few examined primary care settings. ${ }^{6,7}$

A seminal study of primary care practice transformation at Group Health Cooperative reported a large decrease in scores on the Maslach Burnout Inventory (MBI) emotional exhaustion scale among clinicians at a primary care transformation pilot site compared with their counterparts at the organization's other primary care clinics. ${ }^{8,9}$ The study included only a single intervention clinic with a relatively small sample of clinicians and staff. The Healthy Workplace Study was a more recent cluster-randomized trial evaluating interventions at several primary care clinics to reduce 
burnout. ${ }^{10}$ Burnout was measured at baseline and 12 to 18 months later using a single-item self-reported burnout measure. Clinicians at the intervention sites implementing focused transformation interventions were 3 times more likely than those at control clinics to have a decrease in burnout scores. In contrast, a recent observational study of Lean workflow redesign in primary care at the Palo Alto Medical Foundation found that burnout increased during the transformation initiative. ${ }^{11}$ This study measured all $3 \mathrm{MBI}$ burnout scales and perceptions of the work environment among physicians and staff at baseline and between 5 months and 3 years after initiating Lean redesign. Although ratings of overall work satisfaction and engagement among physicians and staff increased, mean scores worsened for physicians on 2 of the 3 burnout scales. Among staff, mean scores worsened on one burnout scale and improved on another. The authors of this study concluded that practice redesign has the potential of "overtaxing an already overstretched workforce," echoing a concern raised by the evaluators of a primary care transformation program sponsored by the American Academy of Family Physicians in 2006-2008. ${ }^{12}$ Although that mixed-methods evaluation did not quantitatively track physician burnout using validated burnout scales, it found that practice reengineering sometimes overwhelms clinicians and staff, aggravating rather than alleviating burnout. A final recent study tracking burnout during primary care transformation documented more neutral outcomes. This investigation of the Comprehensive Primary Care initiative sponsored by the Centers for Medicare \& Medicaid Services measured burnout among physicians in participating and control practices in 2013 and 2016, finding that burnout was similar among physicians at intervention and control practices in both study years, and that burnout neither improved nor worsened at intervention sites during the initiative. ${ }^{13}$ All these studies had relatively brief follow-up times of 3 years or less. To our knowledge, no published study has tracked primary care clinician experience over a longer period of sustained practice transformation.

For the past decade, the San Francisco Health Network (SFHN) and UCSF Health, 2 large health delivery organizations, have been transforming their primary care practices. The UCSF Center for Excellence in Primary Care (CEPC) has collaborated with these organizations in facilitating and evaluating care redesign. Transformation efforts have been multifaceted and varied, but they have adhered to the unifying transformation model of the CEPC's Ten Building Blocks of High Performing Primary Care ${ }^{14}$ and Quadruple Aim ${ }^{15}$ conceptual frameworks that include joy in practice as one of the goals of practice redesign.
In this article, we describe trends in burnout among primary care clinicians and staff measured over 7 years in these 2 health care organizations during a period of practice transformation.

\section{METHODS}

\section{General Approach}

This pragmatic study was conducted as a partnership between the 2 health care organizations and an embedded evaluation team. The principal study goal was to assess the organizations' ongoing efforts in practice transformation using a participatory, learning health system approach ${ }^{16}$ with findings from periodic assessments directly communicated to the practices to inform continuous improvement strategies. We sought to understand whether there was evidence of progress made in implementing practice redesign, and how these efforts were associated with the well-being of clinicians and staff as well as with patient outcomes.

\section{Study Setting}

The study took place in 10 primary care clinics in the SFHN, a county-administered health system, and 7 primary care practices in UCSF Health, a universityadministered health system. The 17 clinics together serve about 150,000 patients. All clinicians and staff are employed by either the county public health department or university, and most nonphysician staff are unionized. The study clinics include an array of family medicine, general internal medicine, geriatric, and interspecialty primary care practices serving a racially and ethnically diverse urban population. The SFHN clinics primarily care for Medicaid, Medicare, and uninsured patients, and the UCSF Health practices for a mix of privately and publicly insured patients. Two of the study practices in each system are large residency program teaching clinics.

\section{Primary Care Transformation}

For the past decade, SFHN and UCSF Health have invested in primary care transformation using the conceptual framework of the Ten Building Blocks. ${ }^{14}$ During the initial years, CEPC and a partnering organization deployed practice coaches at a majority of the study clinics; in the latter years, the health systems institutionalized Lean improvement and management methods to continue to drive improvement work. The specific improvement projects differed across the 2 systems and their individual practices based on system priorities and individual clinic needs, in an iterative and continuous effort to achieve high-performing primary care. Both SFHN and UCSF Health, however, implemented similar transformation elements (Table 1). 
Both also adopted new electronic health record systems in the year before the study began in 2012, with several of the SFHN clinics not going live until 2013. All UCSF Health practices received Patient Centered Medical Home level 3 recognition from the National Committee for Quality Assurance during the study period $_{i}$ the SFHN clinics did not apply for recognition. Both systems participate in the California Medicaid waiver program, which provides substantial financial incentives to public delivery systems for achieving quality improvement targets in primary care.

Both organizations made demonstrable progress in practice transformation during the study period. Annual self-assessments by clinic leaders of Building Block implementation showed steady improvement in ratings for all but 1 Building Block in 1 organization that was already scored very highly at baseline. Survey data from clinicians documented improved perceptions over time of team-based care elements such as team culture, staff involvement in panel management, and support for patients with complex care needs. Both systems achieved all quality performance targets in cancer screening, hypertension control, depression screening, and other metrics to qualify for the maximum possible amount of Medicaid waiver incentives during the final 2 years of the study. Patient experience also improved as measured by standard patient surveys.

\section{Measuring Burnout With Clinician and Staff Surveys}

Beginning in 2012, CEPC has conducted surveys of all primary care clinicians (attending physicians, residents and fellows, nurse practitioners, and physician assistants providing continuity of care to an empaneled group of patients) and care team staff at these clinics. Clinic directors prepare rosters of all active clinicians and staff at their sites that specify the name and occupation of each employee and trainee. All individuals on these rosters are eligible for the surveys, and rosters are updated before each survey wave. Each clinician on the roster receives an e-mail invitation to complete a web-based questionnaire, with up to 5 reminder e-mails to nonrespondents. Surveys of staff were initially administered on paper during staff meetings and migrated over time to web-based versions at most of the practices. Respondents are entered into a $\$ 25$ gift card raffle. Surveys have been administered on an approximately annual basis with a total of 6 survey waves completed between 2012 and 2018. The survey is administered under a study protocol approved by the UCSF Institutional Review Board (protocol 11-08048). Survey data at the clinic and system level (but not individual respondent level) are shared with clinic leaders to inform improvement strategies and track clinician and staff experience.

Survey items include measures of burnout, perceptions of the work environment, and a limited set of respondent characteristics. Burnout measures are the validated 16-item MBI general survey emotional exhaustion and cynicism subscales. Each MBI subscale is composed of 5 symptoms. Respondents rate how often they experience each symptom from 0 (never) to 6 (every day), and responses are summed for each subscale (possible composite score of 0 to 30 points). Higher scores on the emotional exhaustion and cynicism subscales indicate a greater amount of burnout. Based on published standard population distributions, high emotional exhaustion is defined as a score greater than or equal to 16 , and high cynicism as a score greater than or equal to $11 .{ }^{17}$ The MBI subscales measure distinct elements of the experience of burnout, with the emotional exhaustion subscale capturing a sense of work overload and the cynicism subscale capturing a sense of loss of meaning in work. The first 2 survey waves included a third MBI subscale, the personal accomplishment subscale. This subscale was dropped from subsequent surveys to reduce response burden in the survey, and that subscale is not included in this study. Survey items on respondent characteristics were limited to questions about tenure of employment and full- vs part-time work status.
Table 1. Examples of Building Block Elements Implemented by Both the SFHN and UCSF Health

\begin{tabular}{|c|c|}
\hline Building Block Element & Example \\
\hline Engaged leadership & $\begin{array}{l}\text { Primary care service lines led by executive medical and } \\
\text { administrative/nursing directors teams, regular staff and } \\
\text { clinician meetings, Lean management including daily hud- } \\
\text { dles, formal leadership training for some clinic managers }\end{array}$ \\
\hline Data-driven improvement & $\begin{array}{l}\text { Performance dashboards regularly provided at the clinic } \\
\text { and individual PCC levels, transparent posting of dash- } \\
\text { boards in clinics }\end{array}$ \\
\hline Empanelment & $\begin{array}{l}\text { All patients formally empaneled with a PCC, panel sizes } \\
\text { computed for each PCC and adjusted for patient complex- } \\
\text { ity, panel size targets adjusted for clinical FTE effort }\end{array}$ \\
\hline Team-based care & $\begin{array}{l}\text { Behavioral health integration or coordination, scribes for } \\
\text { some PCCs, complex care teams, pharmacists at some sites }\end{array}$ \\
\hline Patient-team partnership & Patient advisory councils at most clinics \\
\hline Population management & $\begin{array}{l}\text { Registries and panel management to close preventive and } \\
\text { chronic care gaps }\end{array}$ \\
\hline Prompt access to care & $\begin{array}{l}\text { Call centers, modified scheduling templates to improve } \\
\text { access, active management of new patient appointment } \\
\text { scheduling }\end{array}$ \\
\hline
\end{tabular}


Because of the desire to reassure respondents that their individual responses would remain confidential when pooled survey data were shared with clinic leaders, the survey did not include items ascertaining age, sex, or race and ethnicity.

\section{Analysis}

Individual clinician and staff survey responses were aggregated at the level of each system for each survey wave and means were computed for the emotional exhaustion and cynicism subscales, with repeated cross-sectional analysis used to measure longitudinal trends at the level of each health system. The proportions of respondents with high levels of emotional exhaustion and cynicism were also calculated. Responses from residents were excluded from the analyses because of the highly variable time they spent in clinic and the unique set of factors affecting burnout among residents. Regression models were constructed stratified by system and type of worker (clinician or staff) to test the statistical significance of time trends, with a linear term for survey year as the predictor variable and $\mathrm{MBI}$ subscales as the outcome variables. Tenure at the clinic and full- vs part-time status were included as covariates. Each respondent was included as a unique observation for each year he or she responded to the survey, with error terms adjusted for repeated measures from the same individual for those who responded to more than one survey wave.

\section{RESULTS}

Response rates across survey waves for the 17 clinics in this analysis ranged from $62 \%$ to $90 \%$ for clinicians and $63 \%$ to $90 \%$ for staff. The number of respondents in each survey wave ranged from 134 to 216 for clinicians and 250 to 405 for staff, reflecting growth in clinic staffing and improving response rates. Table 2 shows the characteristics of respondents to the 2018 survey wave.

\section{Trends in Burnout: SFHN}

For clinicians in the SFHN, mean scores on both the emotional exhaustion and cynicism MBI subscales decreased over time (Figure 1). The decrease over time was statistically significant for the cynicism score $(P=.04)$ and approached significance for emotional exhaustion $(P=.07)$. When subscales were measured as dichotomous outcomes rather than means, the percentage of clinicians with high emotional exhaustion decreased from $56 \%$ in 2012 to $36 \%$ in $2018(P=.02$ for time trend), and the percentage with high cynicism decreased from $25 \%$ to $20 \%$ ( $P=.33$ ) (data not shown).

The pattern in burnout scores among SFHN staff differed from that observed among clinicians. Among staff, mean scores for emotional exhaustion remained relatively stable ( $P=.97$ for time trend), while cynicism scores among staff showed a nonsignificant trend of steadily increasing over time $(P=.27)$. Measured as a dichotomous outcome, the percentage of staff with high emotional exhaustion was $42 \%$ in 2012 and $46 \%$ in $2018(P=.78)$, and the percentage with high cynicism was $26 \%$ in 2012 and $39 \%$ in $2018(P=.04)$.

\section{Trends in Burnout: UCSF Health}

The UCSF Health clinician mean burnout scores started at a lower level than the baseline SFHN scores and, unlike the trends among SFHN clinicians, UCSF Health clinician burnout scores did not steadily decrease over time but had an inverted-U shaped pattern (Figure 2). The mean emotional exhaustion score increased in study year 2014 and then returned by 2018 to a level (14.7) slightly below the baseline 2012 level (15.0). The mean cynicism score also initially increased, with a large jump between 2012 and 2013 from 6.8 to 9.3 , before gradually decreasing to 8.4 in 2018. There were no statistically significant overall time trends for the emotional exhaustion and cynicism scores among UCSF Health clinicians. These patterns were similar when burnout was measured as a dichoto-

Table 2. Survey Respondent Characteristics, 2018

\begin{tabular}{|c|c|c|}
\hline Characteristic & $\begin{array}{l}\text { Clinicians } \\
(n=215)\end{array}$ & $\begin{array}{c}\text { Staff } \\
(n=397)\end{array}$ \\
\hline \multicolumn{3}{|l|}{ System, No. (\%) } \\
\hline SFHN & $112(52.1)$ & $249(62.7)$ \\
\hline UCSF & $103(47.9)$ & $128(37.3)$ \\
\hline \multicolumn{3}{|l|}{ Position, No. (\%) } \\
\hline Physician & $170(80.2)$ & $\ldots$ \\
\hline $\begin{array}{l}\text { Nurse practitioner, physician } \\
\text { assistant }\end{array}$ & $42(19.8)$ & $\ldots$ \\
\hline Nurse (RN or LVN) & $\ldots$ & $84(21.1)$ \\
\hline Medical assistant & $\ldots$ & $123(30.9)$ \\
\hline Front office/clerical & $\ldots$ & $112(28.1)$ \\
\hline Behavioral health & $\ldots$ & $33(8.3)$ \\
\hline Other & $\ldots$ & $46(11.6)$ \\
\hline \multicolumn{3}{|l|}{ Tenure at time of survey, No. (\%) } \\
\hline$<1 \mathrm{y}$ & $10(9.0)$ & $42(17.2)$ \\
\hline $1-5 y$ & $138(64.0)$ & $138(56.6)$ \\
\hline$>5 y$ & $30(27.0)$ & $64(26.2)$ \\
\hline \multicolumn{3}{|l|}{$\begin{array}{l}\text { Patient care sessions per week, } \\
\text { No. (\%) }\end{array}$} \\
\hline 1-2 half-days & $80(37.6)$ & $\ldots$ \\
\hline 3-5 half-days & $87(40.1)$ & $\ldots$ \\
\hline$\geq 6$ half-days & $46(21.6)$ & $\ldots$ \\
\hline \multicolumn{3}{|l|}{ Work status, No. (\%) } \\
\hline Full time ( $>20$ hours/week) & $\ldots$ & $362(94.3)$ \\
\hline Part time (<20 hours/week) & $\ldots$ & $22(5.7)$ \\
\hline
\end{tabular}




\section{Figure 1. Trends in burnout among SFHN clinicians and staff.}



SFHN = San Francisco Health Network

Notes: The figure shows mean emotional exhaustion and cynicism scores by year. Higher scores indicate a higher level of burnout. The $P$ values are from regression models using year as the primary predictor, controlling for tenure of employment and full-vs part-time status at the clinic, and adjusting for clustering of individuals who responded to more than 1 survey wave.

mous outcome; the percentage of clinicians with high emotional exhaustion was $48 \%$ in 2012 and $44 \%$ in $2018(P=.58)$, and the percentage with high cynicism was $16 \%$ in 2012 and $33 \%$ in $2018(P=.32)$.

The pattern for UCSF Health staff was a U-shaped pattern that was the opposite of that observed for clinicians. The staff mean score for emotional exhaustion decreased from 14.7 in 2012 to 12.1 in 2014. In the years after, however, emotional exhaustion increased to 13.6 in 2018 , negating any overall trend $(P=.95$ for overall time trend). There was a similar pattern for cynicism scores over time among UCSF Health staff, with a decrease from 8.4 in 2012 to 6.2 in 2014, then an increase back to 7.6 in $2018(P=.94)$. The percentage of staff with high emotional exhaustion was $45 \%$ in 2012 and $43 \%$ in $2018(P=.70)$, and with high cynicism was $24 \%$ in 2012 and $29 \%$ in $2018(P=.60)$.

\section{DISCUSSION}

This study is to our knowledge the first to report trends over this length of an observation period in scores on validated burnout scales among primary care practices engaged in transformation work. Our results do not tell a simple story; trends in burnout differed between the 2 systems and between clinicians and staff. We interpret our findings as supporting the following trio of conclusions.

First, both health care organizations made meaningful strides in practice transformation. Triangulation of data from clinic leader self-assessments, surveys of clinicians and staff, and clinical performance measures supports the conclusion that clinics in both organizations made progress in implementing the 10 Building Blocks of High Performing Primary Care and made gains in quality of care and patient experience. 


\section{Figure 2. Trends in burnout among UCSF Health clinicians and staff.}

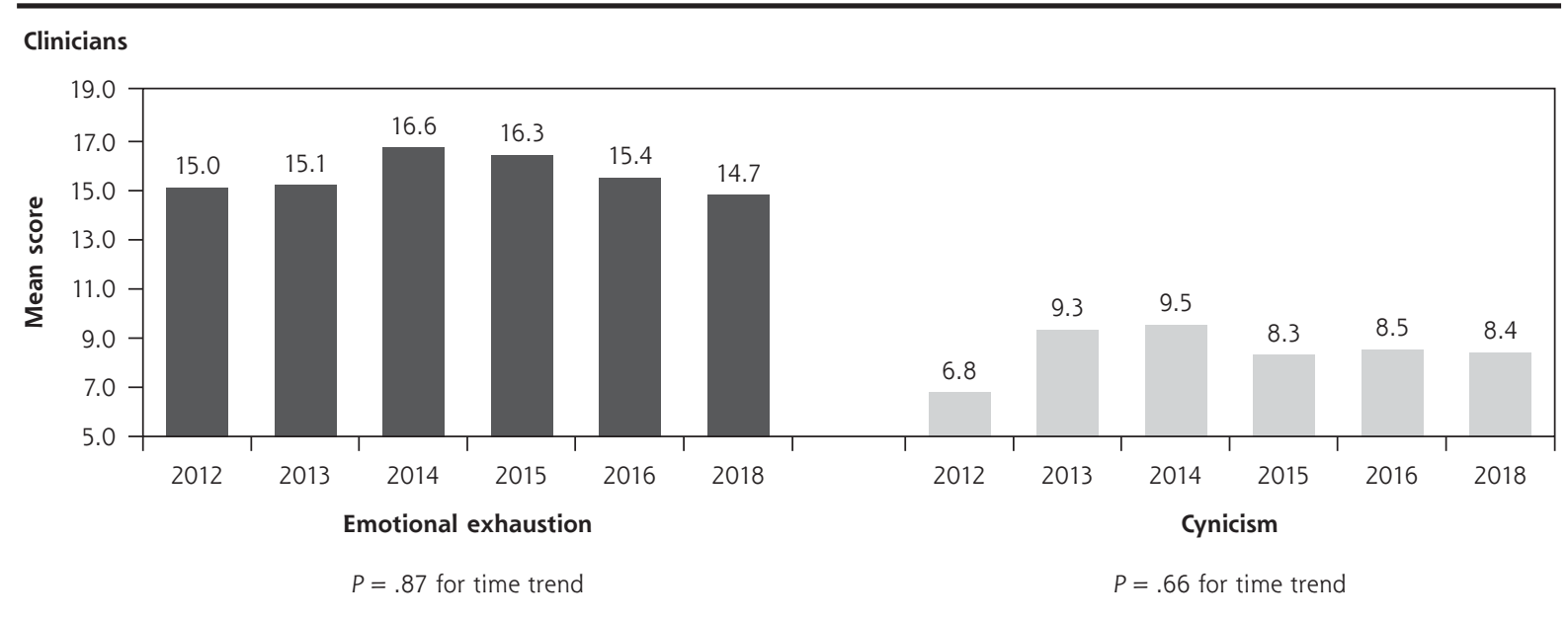

Staff

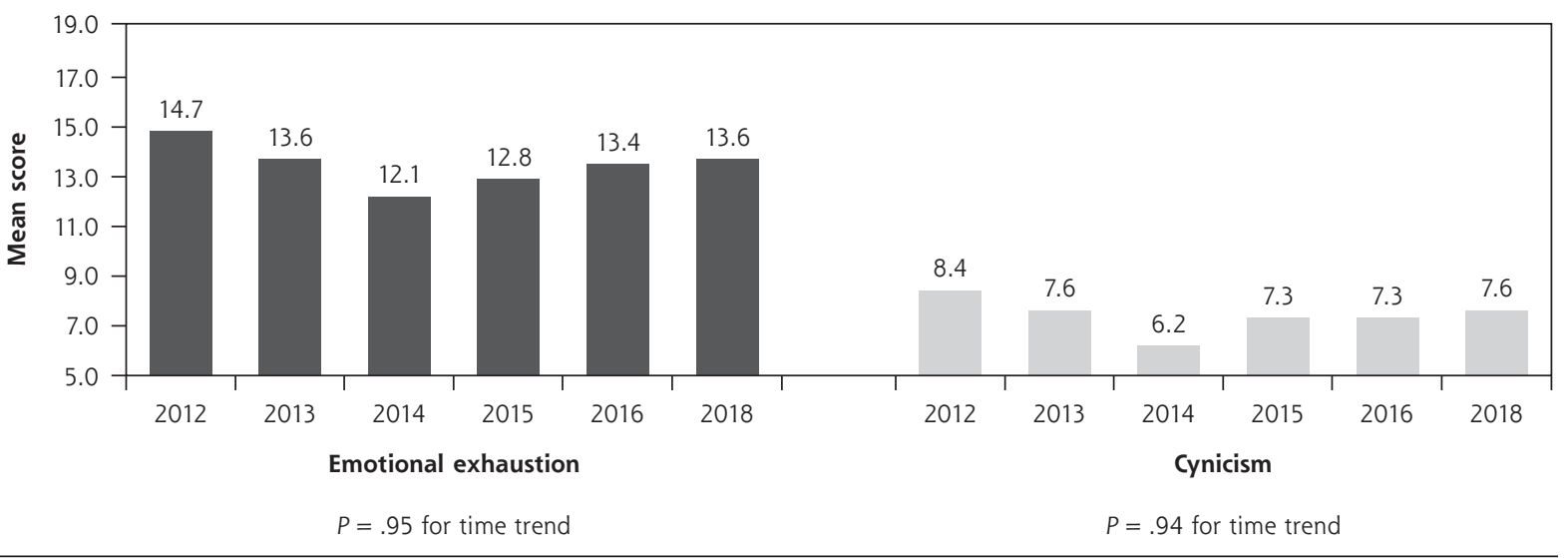

UCSF = University of California San Francisco.

Notes: The figure shows mean emotional exhaustion and cynicism scores by year. Higher scores indicate a higher level of burnout. The $P$ values are from regression models using year as the primary predictor, controlling for tenure of employment and full vs part time status at the clinic and adjusting for clustering of individuals who responded to more than 1 survey wave.

Second, the divergent trends in clinician burnout scores between the SFHN and UCSF Health despite shared progress on practice transformation guided by a common conceptual model suggest that clinicians' experience of practice transformation may be highly context dependent. Practice transformation in the SFHN was associated with a steady decrease in emotional exhaustion and cynicism among clinicians. In contrast, among UCSF Health clinicians, burnout initially worsened and then decreased back toward baseline levels. Research on practice transformation and organizational change has highlighted the importance of context for explaining the highly variable patterns observed across studies. ${ }^{18-20}$ Although both the SFHN and UCSF Health are major teaching institutions for UCSF trainees, they have different cultures and characteristics that may have influenced the different patterns in burnout we observed. Virtually all UCSF Health physicians are faculty members, whereas SFHN physicians are a mix of UCSF faculty members and Department of Public Health-employed physicians. UCSF Health is nested in a large academic health center with a traditional emphasis on tertiary and quaternary care, whereas the SFHN is a safety-net system with a greater emphasis on community-based primary care. Differences in these and related system characteristics may have influenced the divergent patterns in clinician burnout trends. Another contextual factor may be differences in starting points for the transformation journey. When measured at the first study year, SFHN clinic leader self-assessments of Building Block implementation were higher than the baseline UCSF Health scores on these measures, suggesting that the SFHN was further along the transformation path than was UCSF Health at the 
start of the study. To the extent that the initial increase in burnout scores among UCSF Health clinicians reflects a disruptive early phase of practice transformation, our study may have missed a similar disruptive phase that possibly occurred in the SFHN before the first survey was fielded. Just as these factors may have influenced the patterns we observed in the SFHN and UCSF Health, contextual factors also likely explain some of the differences in results across previously published longitudinal studies. For example, although the positive burnout outcomes for a single prototype clinic at Group Health Cooperative was mirrored by the outcomes for the early adopter clinics at Palo Alto Medical Foundation, ${ }^{11}$ in the latter system, the positive trends for the early adopter clinics were offset by the negative trends at the more numerous later-adopting clinics.

Third, clinicians and staff working in the same systems may have very different experiences of the transformation process. Practice changes that reduce clinician burnout may not reduce-and may potentially worsen-burnout among staff. Burnout trends among staff did not follow the same trends for clinicians in the same system, and if anything, moved in the opposite direction from clinician scores. Most primary care transformation models emphasize teambased care with expanded staff roles and sharing of tasks such as panel management. ${ }^{21}$ Although some staff may welcome these changes as an enhancement of their professional roles, others may experience new team models as a stressful increase in workload. These divergent trends among clinicians and staff were also observed to a degree in the study at Palo Alto Medical Foundation, ${ }^{11}$ although in that setting trends in staff experiences were somewhat better than those for clinicians. Burnout measures and the experience of burnout may also have different properties among staff than clinicians. In our previous studies using data from these surveys, we found that, among individuals reporting a strong sense of team culture, working in tighter "teamlet" structures had a cross-sectional association with lower emotional exhaustion among clinicians but not staff. ${ }^{3}$ We also found that burnout predicted subsequent job turnover among clinicians but not staff in these same 2 organizations. ${ }^{22}$ Although we do not conclude from the divergent trends in clinician and staff burnout in our current study that transformation is a "zero sum game" and that practice changes that reduce burnout among one group necessarily increase it among the other, we consider our finding to be an important reminder that interventions to promote joy in practice must consider the well-being of all team members. Improvement initiatives and burnout research will need to consider staffing levels, staff training, culture change, and related factors that facili- tate team models being implemented in a manner that avoids the risk of aggravating staff burnout.

Our study has several strengths, including the longitudinal design, use of validated burnout measures, inclusion of staff as well as clinicians, and the high response rate. It also has several limitations. The study setting was 2 relatively large integrated health care organizations with employed physicians in a single region, with several of the largest clinics serving as teaching sites with many physicians at those sites having part-time clinical practices. Our findings may not be generalizable to other types of practice settings. Although our pragmatic, learning health system approach has advantages in allowing us to study over several years the real-world experience of 2 systems implementing practice transformation, it limits our ability to make causal inferences about factors that might explain patterns in burnout outcomes across clinics and systems. Transformation interventions were heterogeneous with variable dosing across clinics depending on factors such as clinic readiness, changes in clinic personnel, leadership engagement, and availability of resources. The relatively high turnover of clinicians and staff precluded analyzing longitudinal data for the same individuals for the duration of the study. We measured a limited set of potential confounders at the individual respondent level, and other unmeasured confounders might influence some of the outcomes. The study was not funded at a level that would have allowed us to perform rigorous qualitative research to explore explanatory factors more thoroughly. The causes of burnout are multifactorial, including influences exogenous to health systems such as the growing unaffordability of housing in the San Francisco region.

In summary, our study suggests that practice transformation can be associated with reductions in burnout among clinicians-but that progress in reducing burnout may depend on the context of the specific system and practice settings and, in some contexts, burnout may get worse before getting better. It also sounds a note of caution that the movement to promote joy in practice must ensure that transformation reduces burnout among staff as well as clinicians. We have found it valuable to include regular measurement of work experience to assess progress on the quadruple aim in the SFHN and UCSF Health. Measuring and responding to burnout levels sends a message to clinicians and staff that their well-being matters to leaders. We encourage other organizations to integrate measures of clinician and staff experience into their practice improvement dashboards. Primary care practice transformation is a journey, not a destination, ${ }^{23}$ and one that requires continuing efforts to promote meaningful work and sustainable workloads among all members of the primary care team. 
To read or post commentaries in response to this article, see it online at http://www.AnnFamMed.org/content/17/Suppl_1/S9.

Key words: primary care; burnout; mental health; professional practice; practice improvement; organizational change; practice-based research

Submitted August 30, 2018; submitted, revised, November 16, 2018; accepted December 21, 2018.

Funding support: This project was supported in part by a grant from the Metta Fund in partnership with the San Francisco Health Plan. The San Francisco Health Network and UCSF Health also funded data collection as part of ongoing practice improvement efforts.

Disclaimer: The Metta Fund had no role in interpretation of the data or writing of the manuscript.

Acknowledgments: The authors thank Kate Dubé for her contributions to this project, and the clinicians, staff, and administrators of the primary care clinics at SFHN and UCSF Health for their partnership in this work.

\section{References}

1. Shanafelt TD, Hasan O, Dyrbye LN, et al. Changes in burnout and satisfaction with work-life balance in physicians and the general US working population between 2011 and 2014. Mayo Clin Proc. 2015; 90(12):1600-1613.

2. Linzer M, Manwell LB, Williams ES, et al; MEMO (Minimizing Error, Maximizing Outcome) Investigators. Working conditions in primary care: physician reactions and care quality. Ann Intern Med. 2009; 151(1):28-36, W6-W9.

3. Willard-Grace R, Hessler D, Rogers E, Dubé K, Bodenheimer T, Grumbach K. Team structure and culture are associated with lower burnout in primary care. J Am Board Fam Med. 2014;27(2):229-238.

4. De Marchis EH, Knox M, Hessler D, et al. Physician burnout and higher clinic capacity to address patients' social needs. J Am Board Fam Med. 2019;32(1):69-78.

5. Rassolian M, Peterson LE, Fang B, et al. Workplace factors associated with burnout of family physicians. JAMA Intern Med. 2017; 177(7):1036-1038.

6. West CP, Dyrbye LN, Erwin PJ, Shanafelt TD. Interventions to prevent and reduce physician burnout: a systematic review and metaanalysis. Lancet. 2016;388(10057):2272-2281.

7. Panagioti $M$, Panagopoulou E, Bower $P$, et al. Controlled interventions to reduce burnout in physicians: a systematic review and meta-analysis. JAMA Intern Med. 2016;177(2):195-205.
8. Reid RJ, Fishman PA, Yu O, et al. Patient-centered medical home demonstration: a prospective, quasi-experimental, before and after evaluation. Am J Manag Care. 2009;15(9):e71-e87.

9. Reid RJ, Coleman K, Johnson EA, et al. The Group Health medical home at year two: cost savings, higher patient satisfaction, and less burnout for providers. Health Aff (Millwood). 2010;29(5):835-843.

10. Linzer M, Poplau S, Grossman E, et al. A cluster randomized trial of interventions to improve work conditions and clinician burnout in primary care: results from the Healthy Work Place (HWP) study. J Gen Intern Med. 2015;30(8):1105-1111.

11. Hung DY, Harrison MI, Truong Q, Du X. Experiences of primary care physicians and staff following lean workflow redesign. BMC Health Serv Res. 2018;18(1):274.

12. Nutting PA, Crabtree BF, Miller WL, Stange KC, Stewart E, Jaén C. Transforming physician practices to patient-centered medical homes: lessons from the National Demonstration Project. Health Aff (Millwood). 2011;30(3):439-445.

13. Peikes DN, Swankoski K, Hoag SD, et al. The effects of a primary care transformation initiative on primary care physician burnout and workplace experience. J Gen Intern Med. 2019;34(1):49-57.

14. Bodenheimer T, Ghorob A, Willard-Grace R, Grumbach K. The 10 building blocks of high-performing primary care. Ann Fam Med. 2014;12(2):166-171.

15. Bodenheimer T, Sinsky C. From triple to quadruple aim: care of the patient requires care of the provider. Ann Fam Med. 2014;12(6): 573-576.

16. Grumbach K, Lucey CR, Johnston SC. Transforming from centers of learning to learning health systems: the challenge for academic health centers. JAMA. 2014;311(11):1109-1110.

17. Maslach C, Jackson S, Leiter M. MBI: Maslach Burnout Inventory Manual. 3rd ed. Palo Alto, CA: Consulting Psychologists Press; 1996.

18. Tomoaia-Cotisel A, Scammon DL, Waitzman NJ, et al. Context matters: the experience of 14 research teams in systematically reporting contextual factors important for practice change. Ann Fam Med. 2013;11(Suppl 1):S115-S123.

19. Greenhalgh T, Robert G, Macfarlane F, Bate P, Kyriakidou O. Diffusion of innovations in service organizations: systematic review and recommendations. Milbank Q. 2004;82(4):581-629.

20. Hung D, Gray C, Martinez M, Schmittdiel J, Harrison MI. Acceptance of lean redesigns in primary care: a contextual analysis. Health Care Manage Rev. 2017;42(3):203-212.

21. Ghorob A, Bodenheimer T. Share the Care: building teams in primary care practices. J Am Board Fam Med. 2012;25(2):143-145.

22. Willard-Grace R, Knox M, Huang B, et al. Burnout and health care workforce turnover. Ann Fam Med. 2019;17(1):36-41

23. McNellis RJ, Genevro JL, Meyers DS. Lessons learned from the study of primary care transformation. Ann Fam Med. 2013;11(S1):S1-S5. 